\title{
Mobilidade socio-ocupacional no Brasil: novo procedimento para delimittação dos estratos ocupacionais e análise dos dados da PNAD de 2014
}

\author{
Camilla Oliveira* \\ Rodolfo Hoffmann* (])
}

\section{Resumo}

O objetivo deste trabalho é analisar, a partir dos dados da PNAD de 2014, a mobilidade socio-ocupacional inter e intrageracional na sociedade brasileira e a influência da migração e da cor dos indivíduos no processo de mobilidade social. É proposta uma nova metodologia de estratificação para a construção de estratos socio-ocupacionais, utilizando características como educação, renda e ocupação dos indivíduos. Ainda que marcada por deslocamentos de curta distância entre os estratos socio-ocupacionais, a mobilidade social observada nos dados coletados em 2014 é marcada por chances de mobilidade ascendente, o que denota certa dinamicidade na sociedade brasileira. A análise revela um esvaziamento dos estratos baixos em favor dos estratos médios quando se compara a distribuição socio-ocupacional dos ocupados em 2014 com a geração dos pais. Há uma predominância na mobilidade circular, indicando que os movimentos realizados na estrutura socio-ocupacional se deram mais pela substituição e trocas em postos de trabalho já existentes do que pela transformação da estrutura ocupacional. Foram encontradas evidências de que indivíduos migrantes apresentam mobilidade superior aos não migrantes. Todavia, indivíduos pretos e pardos encontram condições desiguais de mobilidade socio-ocupacional quando comparados aos brancos, e essa desigualdade é atenuada quando se consideram os indivíduos migrantes.

Palavras-chave: mobilidade socio-ocupacional, estratificação social, migração, cor.

* Escola Superior de Agricultura Luiz de Queiroz, Universidade de São Paulo, Piracicaba, SP, Brasil. 


\section{Socio-occupational mobility in Brazil: new occupational stratification procedure and analysis of data from PNAD/2014}

\section{Abstract}

The objective of the present study is to analyze the intra- and intergenerational socio-occupational mobility in Brazil and the influence of migration and skin color in the person's social mobility, using data from a national household survey (PNAD) collected in 2014. We propose a new stratification procedure for constructing socio-occupational strata using individuals' characteristics such as education, income and occupation. Although characterized by short displacements between socio-occupational strata, the social mobility observed in the 2014 data shows chances of upward mobility, indicating certain dynamism in the Brazilian society. Comparing the socio-occupational distribution of workers in 2014 with their father's distribution, the results show the weakening of lower strata in favor of medium strata. A predominance of circular mobility was observed, indicating that the movements in the socio-occupational structure occurred by substitution and changes in the already existing job positions rather than by the transformation of the occupational structure. The results show that migrant individuals presented higher mobility in relation to non-migrants. However, black and brown persons obtain less upward mobility when compared with white ones, and such difference is smaller among the migrants.

Keywords: socio-occupational mobility, social stratification, migration, skin color.

\section{Introdução}

mobilidade social é o fenômeno que reflete o movimento dos
agentes em dada estrutura social. O seu estudo busca identificar
a direção dos deslocamentos em um determinado período, sua magnitude e as variáveis que influenciam o processo.

A necessidade de uma escala que hierarquize os agentes se traduz na forte ligação que existe entre o estudo da mobilidade social e o estudo da estratificação social (Hertel, 2017). Os critérios para a construção da estratificação social, por meio da qual se estuda a mobilidade, são escolhidos a partir da abordagem que se pretende utilizar para medir a desigualdade 
de oportunidades na sociedade, e dependem também da disponibilidade das informações. Em geral, utiliza-se um ou mais critérios, como, por exemplo, educação, renda e ocupação.

A mobilidade social pode ser vista sob duas perspectivas, a intergeracional e a intrageracional. Do ponto de vista metodológico, as análises concentramse nas transições experimentadas pelos indivíduos entre dois momentos de referência, que podem ser denominados de origem e destino. No caso da mobilidade intergeracional, em que o interesse reside na dinâmica social entre diferentes gerações, é usual confrontar a ocupação atual do indivíduo (destino) com a ocupação do pai no início da vida profissional do indivíduo (origem). No caso da mobilidade intrageracional, em que a intenção é identificar as mudanças conquistadas pelo indivíduo na sua geração, contrasta-se a ocupação atual do indivíduo (destino) com a ocupação que ele tinha no início da carreira profissional (origem).

Os movimentos podem ser ascendentes, caso a posição de destino seja melhor que a de origem, ou descendentes, caso a posição de destino seja pior que a de origem. A permanência na posição inicial caracteriza imobilidade. A avaliação da mobilidade também pode ser realizada por meio dos conceitos de mobilidade circular e mobilidade estrutural (Pastore, 1979). A mobilidade estrutural corresponde aos movimentos que aconteceram como resultado de mudanças na estrutura socio-ocupacional e criação de novas oportunidades no mercado de trabalho. A mobilidade circular, por outro lado, ocorre caso a mobilidade se dê por trocas de posições, substituições ou aposentadorias.

Quando consideramos a forma de mensurar a mobilidade, há na literatura quatro gerações metodológicas. A primeira geração utiliza matrizes de transição e índices de mobilidade (Glass; Hall, 1954; Hutchinson, 1960; Rogoff, 1953); na segunda geração há maior preocupação com a descrição do processo que origina o fenômeno a partir do uso de análises de trajetórias (Blau; Duncan, 1967); a terceira inova utilizando modelos log-lineares na tentativa de solucionar alguns problemas metodológicos discutidos na literatura (Goldthorpe et al., 1982; Goodman, 1979; Wright, 1979); e, 
por fim, a quarta geração faz análises tentando conciliar as duas gerações anteriores (DiPrete, 1990; Breen, 1994; Hendrickx; Ganzeboom, 1998).

Como salienta Silva (1999), o estudo da mobilidade social brasileira é bastante deficiente. Nos últimos anos ocorreu uma renovação do interesse pelo tema, com maior participação de economistas nos novos trabalhos realizados. Entretanto, a metodologia usada na tradição da sociologia, com construção das matrizes de mobilidade socio-ocupacional, permite uma análise mais aprofundada da dinâmica dos movimentos. Na literatura brasileira, cabe destacar os trabalhos de Pastore (1979), Scalon (1999), Pastore e Silva (2000) e Ribeiro (2007; 2014; 2017).

O diagnóstico desses trabalhos para o Brasil sugere que houve um alargamento do estrato médio da estrutura social. Esse alargamento foi influenciado, principalmente, por novas oportunidades no mercado de trabalho em ocupações urbanas de baixa remuneração e especialização. Até a década de 1980, a mobilidade social brasileira era alta e apresentava uma grande tendência de ascensão social, como resultado da transformação do país de sociedade predominantemente rural em urbana. Após esse período, apesar da manutenção de altos índices, a mobilidade brasileira passou a apresentar aumento nos níveis de mobilidade descendente e diminuição nos movimentos ascendentes.

Ribeiro (2017) analisa os dados dos suplementos de mobilidade socio-ocupacional da PNAD, disponibilizados nos anos de 1973, 1982, 1988, 1996 e 2014. A partir da aplicação do esquema EGP de classes, proposto por Erikson, Goldthorpe e Portocarero (1979) e usando modelos log-lineares, o autor examina a evolução da mobilidade intergeracional brasileira. Considerando nove classes de status ocupacional, ele mostra que a mobilidade total cresceu de 54\%, em 1973, para 66\%, em 1996, e $67 \%$ em 2014. Considerando apenas três grandes classes, ele verifica que a mobilidade ascendente cresceu de 25\%, em 1973, para 32\%, em 1996, e 34\% em 2014, com a descendente passando de 6\%, em 1973, para 9\%, em 1996, e 11\% em 2014. Ribeiro (2017) conclui que, no período analisado, 
houve diminuição das desigualdades de oportunidades e a mobilidade intergeracional se tornou mais fluida.

Este artigo, seguindo a corrente funcionalista da estratificação social, na qual o papel do indivíduo na sociedade é reflexo das atividades que este desempenha (Davis, 1949; Davis; Moore, 1945; Parsons, 1954), realiza uma análise da mobilidade brasileira com base nas ocupações dos seus agentes, portanto, da mobilidade socio-ocupacional.

Com base nos dados da PNAD de 2014, disponíveis no site do Instituto Brasileiro de Geografia e Estatística (IBGE), propõe-se uma nova metodologia para a construção de uma escala hierarquizada das ocupações brasileiras com base em características socioeconômicas dos indivíduos, como educação, renda e ocupação, a exemplo do trabalho de Blau e Duncan (1967). A partir da escala construída, elabora-se uma análise da mobilidade socioocupacional intergeracional e intrageracional dos indivíduos ocupados, com idade de 16 até 65 anos, por meio de matrizes de transição de status (Hout, 1983) e cálculo de medidas de mobilidade (Yasuda, 1964). As mulheres serão incluídas nas amostras, seguindo uma perspectiva individualista na análise (Hayes; Miller, 1993; Payne; Abbott, 1990; Rose; Marshall, 1988). Analisa-se, ainda, por meio de regressões múltiplas, o efeito da migração e da cor dos indivíduos sobre a mobilidade experimentada por eles.

A expectativa de mobilidade ascendente é uma grande motivação para a migração geográfica e a literatura sobre o tema é bastante vasta. Destacam-se o trabalho precursor no Brasil realizado por Jannuzzi (2000), a respeito da migração e mobilidade social no estado de São Paulo nas décadas de 1980 e 1990, e outros mais recentes (Abramitzky et al., 2019; Major; Machin, 2018; Jannuzzi; Montagner; Taira, 2019). Pastore e Silva (2000, p.92) dedicam um capítulo do livro ao tema "cor e mobilidade ocupacional", concluindo que "os não brancos estão expostos a chances menores de ascensão social; as dificuldades para ascender aumentam junto com o nível do estrato de origem; e os nascidos nos estratos mais elevados estão expostos a riscos maiores de mobilidade descendente". Jesus (2020) apresenta ampla revisão da literatura sobre a relação entre cor e migração e 
o rendimento das pessoas. Com base em dados da PNAD de 1995 a 2015, o autor observa que, no mercado de trabalho brasileiro, "relativamente aos seus respectivos pares não migrantes, tanto os negros quanto os brancos migrantes apresentam melhores resultados e que a diferença se reduziu ao longo do período analisado"; conclui, ainda, que, proporcionalmente, "essa diferença de rendimentos entre migrantes e não migrantes se mostra maior quando se consideram os negros vis-à-vis os brancos." (Jesus, 2020, p. 128). Mas não encontramos na literatura examinada estimativas dos efeitos de migração e cor na mudança intergeracional ou intrageracional em uma medida de status socioeconômico, como será feito aqui.

\section{Metodologia e primeiros resultados}

A escala ocupacional construída para este estudo revisita e modifica a metodologia proposta por Silva (1973), utilizada por Pastore (1979), Pastore e Silva (2000) e Ueda (2001). A proposta é aperfeiçoar a metodologia, usando dados mais recentes para analisar a relação entre renda e escolaridade e adotando um critério estatístico objetivo para delimitar os seis estratos socio-ocupacionais. Evita-se, portanto, a abordagem de prestígio social das ocupações, dadas as dificuldades da mensuração de variáveis subjetivas intrínsecas à noção de "prestígio" e a ausência dessas variáveis nos dados da PNAD.

Foram selecionados indivíduos ocupados com idade de 16 a 65 anos. No processo de tratamento da amostra, foram aplicados filtros e os indivíduos que não apresentavam informações referentes aos anos de escolaridade foram excluídos da análise.

As relações entre renda, ocupação e anos de escolaridade foram analisadas controlando os efeitos da idade do indivíduo. A PNAD de 2014 adota a Classificação Brasileira de Ocupações Domiciliar (CBO-Domiciliar/ IBGE), que lista 511 ocupações das quais 483 estão representadas na pesquisa.

Consideraram-se tanto o rendimento mensal da atividade principal como o número de horas trabalhadas semanalmente. Ao realizar a 
padronização das variáveis para uma semana de 40 horas de trabalho, encontramos observações discrepantes que, após investigação, evidenciaram a necessidade de controle da variável referente às horas trabalhadas. Havia indivíduos declarando ter trabalhado apenas uma hora na semana, apresentando rendimentos mensais padronizados superiores a um milhão de reais. Então foram mantidos na amostra apenas os indivíduos que declararam ter trabalhado de seis a 98 horas por semana.

A variável escolaridade foi medida em anos de estudo, indo de 0 , para nenhuma escolaridade, a 14, para 14 anos, e atribuindo valor 17 para os com escolaridade igual ou superior a 15 anos. A variável idade, utilizada como controle, foi base para classificar as pessoas em dez faixas, com intervalo de cinco anos cada: 16 a 20 anos, 21 a 25, 26 a 30, 31 a 35, 36 a 40, 41 a 45, 46 a 50, 51 a 55, 56 a 60 e 61 a 65.

A amostra final para a construção da estratificação, obtida da PNAD de 2014, conta com 152.940 indivíduos, dos quais 58\% são homens e $42 \%$ mulheres. A amostra, considerando o fator de expansão, representa uma população de 85.644 .380 pessoas. Predominam os indivíduos com idade de 21 a 40 anos (51,7\%) e aqueles com onze anos de escolaridade $(30,9 \%)$, seguidos por aqueles que estudaram quinze ou mais anos $(14,2 \%)$.

Veremos que a medida de status socioeconômico é obtida levando em consideração o rendimento obtido pela pessoa na sua ocupação e sua escolaridade. Mas não podemos, obviamente, somar variáveis com unidades de medidas diferentes (reais e anos de escolaridade). Assim, o próximo passo foi expressar a escolaridade em unidades monetárias por meio de uma equação de rendimentos, controlando o efeito das faixas de idade. Foram ajustadas, para cada uma das dez faixas de idade, equações de rendimento onde a variável dependente $Y$ representa o logaritmo neperiano do rendimento do trabalho principal padronizado pelo tempo semanal de trabalho.

A primeira tentativa segue o procedimento proposto por Silva (1973): realizou-se a estimação de regressões múltiplas para cada faixa de idade, de Y contra 15 variáveis binárias que distinguem os 16 níveis de escolaridade. 
Conforme ilustra a Figura 1, as linhas tendem a ficar em posição mais elevada com idade maior, mas há muita irregularidade.

Figura 1 - Relação entre o logaritmo do rendimento e anos de escolaridade por faixas de idade para 2014, usando 15 variáveis binárias para distinguir os 16 níveis de escolaridade

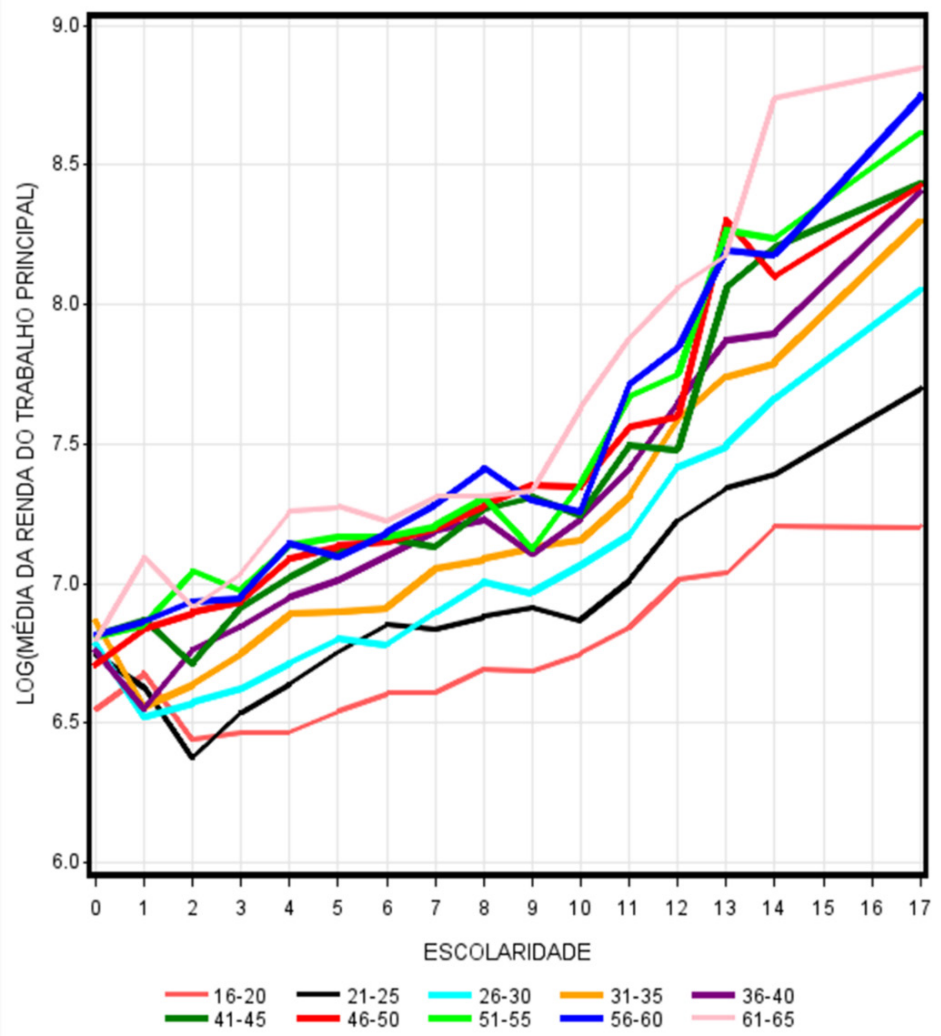

Fonte: Elaborado pelos autores, a partir dos microdados do IBGE.

A análise das linhas na Figura 1 sugere uma mudança de inclinação quando a escolaridade atinge dez anos. Optou-se, então, pela utilização 
de um modelo mais parcimonioso, admitindo uma relação poligonal, com vértice em ponto com abcissa igual a 10, entre o logaritmo da renda e a escolaridade. O gráfico representado na Figura 2 mostra a nova relação, onde é possível observar que, de fato, o retorno da escolaridade tende a ser maior a partir de 10 anos de estudo, corroborando resultados anteriores (Hoffmann; Simão, 2005b; Salvato; Silva, 2008). Seja E a escolaridade (em anos) e seja Z uma variável binária que assume valor zero se a escolaridade (E) for menor ou igual a dez anos e valor 1, em caso contrário. Então, a equação da poligonal para cada faixa de idade é:

$$
Y=\alpha+\beta_{1} E+\beta_{2} Z(E-10)+u
$$

Definindo nove variáveis binárias (, com $\mathrm{h}=1, \ldots, 9$ ) para distinguir as dez faixas de idade, numeradas de 0 a 9 , fazendo $W h=1$ na h-ésima faixa e $\mathrm{Wh}=0$ nas demais, é possível, então, utilizar um modelo de regressão para estimar, simultaneamente, as 10 linhas poligonais (uma para cada faixa de idade):

$$
\begin{aligned}
Y=\alpha+\beta_{1} E+ & \beta_{2} Z(E-10)+\sum_{h=1}^{y} \gamma_{h} W_{h}+\sum_{h=1}^{y} \delta_{h} W_{h} E+ \\
& +\sum_{h=1}^{9} \varphi_{h} W_{h} Z(E-10)+u
\end{aligned}
$$

O antilogaritmo do valor de estimado com base nesse modelo reflete, para cada pessoa, o seu nível de escolaridade, em termos monetários. Dispondo, agora, do rendimento da pessoa na sua ocupação e de uma medida que representa o valor monetário de sua escolaridade, calcula-se a média aritmética dos dois valores, que é o seu Status Socioeconômico Individual (SSEI). 
Figura 2 - Logaritmo da renda estimada pela poligonal em função dos anos de escolaridade por faixas de idade para 2014

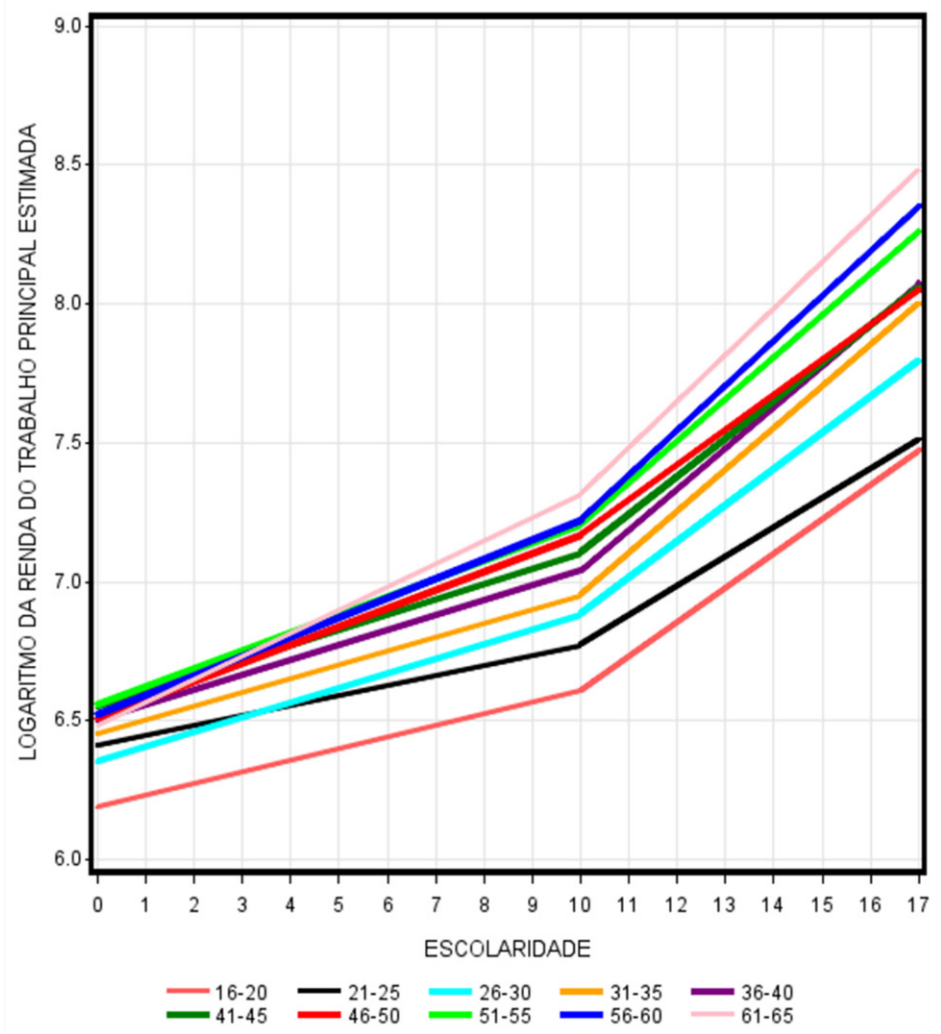

Fonte: Elaborado pelos autores, a partir dos microdados do IBGE.

Calculando a média entre os SSEI de todas as pessoas que possuem a mesma ocupação, obtemos o Status Socioeconômico (SSE) de cada ocupação representada na PNAD 2014. Os SSE foram padronizados para que os escores das ocupações ficassem dentro do intervalo de 0 a $100 .{ }^{1} \mathrm{O}$

1 Para isso os valores foram divididos por 11.197, conforme Oliveira (2018), em análise de dados das PNADs de 1996 e 2014. 
último passo envolve definir os limites dos estratos socio-ocupacionais. Este estudo mantém o número de estratos (seis) e a nomenclatura utilizados no trabalho de Pastore (1979), mas modifica o procedimento para delimitar os seis estratos.

Há na literatura uma arbitrariedade muito grande na definição dos limites que separam os diferentes estratos. Para contornar essa limitação, adotamos a metodologia proposta por Aghevli e Mehran (1981), Davies e Shorroks (1989) e Hoffmann (2005a) para delimitar estratos em distribuições de renda. A metodologia consiste em minimizar a desigualdade intragrupos e maximizar a desigualdade entre grupos na distribuição. Para dois estratos, adotando o Índice de Gini como medida de desigualdade, a divisão ótima é aquela em que o limite entre os dois estratos corresponde ao SSE médio dos dois estratos em conjunto. Para mais de dois estratos, a determinação dos limites entre estratos exige um processo iterativo e detalhes adicionais podem ser encontrados nos trabalhos citados. Os limites encontrados para a estratificação em seis estratos socio-ocupacionais e a distribuição dos indivíduos entre eles serão apresentados nos resultados deste trabalho.

Obtidos os escores de SSE das ocupações para o Brasil em 2014, parte-se para a análise da mobilidade socio-ocupacional. Para essa análise, a amostra foi limitada aos indivíduos que responderam ao suplemento de mobilidade socio-ocupacional e apresentaram informações a respeito da sua primeira ocupação, educação e a ocupação dos seus pais quando o respondente tinha 15 anos de idade. São 22.918 indivíduos que, considerando os fatores de expansão da subamostra, representam uma população de 59.673 milhares de pessoas. As mulheres representam 41,8\% da população, enquanto os homens, 58,2\%.

Uma vez definidos os estratos socio-ocupacionais, construímos as tabelas de contingência, ou matrizes de transição de status, que auxiliam na análise dos movimentos dos indivíduos entre os estratos. A abordagem levou em consideração os trabalhos sociológicos desenvolvidos para a sociedade brasileira (Pastore, 1979; Pastore; Silva, 2000; Ribeiro, 2007) e os dados disponíveis na PNAD de 2014. 
Uma matriz de transição de status $(M)$ tem formato $k \times k$, sendo $k$ o número de estratos adotados; neste estudo $\mathrm{k}=6$. A matriz irá relacionar um status de origem a um status de destino (Hout, 1983). Na análise intergeracional a origem é o status da ocupação do pai quando o indivíduo tinha 15 anos e o ponto de destino é o status da ocupação do indivíduo em 2014; na intrageracional a origem passa a ser a primeira ocupação do próprio indivíduo e o destino se mantém o mesmo.

Para facilitar a explicação da metodologia, adiantamos a apresentação de parte dos resultados, mostrando, na tabela 1 , a matriz de transição de seis estratos obtida para a mobilidade socio-ocupacional intergeracional do Brasil em 2014, em termos populacionais. Pelas linhas é possível fazer uma análise origem-destino, observando para onde se moveram as pessoas com origem em um status específico. Uma coluna mostra a origem dos indivíduos que compõem determinado estrato em 2014. Os elementos da diagonal principal representam os indivíduos imóveis. Abaixo da diagonal principal estão os indivíduos que experimentaram mobilidade ascendente, e acima, mobilidade descendente.

Tabela 1 - Matriz de transição intergeracional brasileira para o ano de 2014/Milhares de pessoas

\begin{tabular}{|c|c|c|c|c|c|c|c|}
\hline \multirow{2}{*}{ STATUS DO PAI } & \multicolumn{6}{|c|}{ STATUS DO FILHO EM 2014} & \multirow{2}{*}{$\begin{array}{c}\text { TOTAL } \\
\text { DOS PAIS }\end{array}$} \\
\hline & 1 & 2 & 3 & 4 & 5 & 6 & \\
\hline 1. Alto & 793 & 557 & 322 & 115 & 262 & 59 & 2.108 \\
\hline 2. Médio-Superior & 794 & 1.250 & 780 & 476 & 759 & 369 & 4.428 \\
\hline 3. Médio-Médio & 492 & 963 & 1.047 & 735 & 1.083 & 653 & 4.973 \\
\hline 4. Médio-Inferior & 624 & 1.379 & 1.721 & 2.081 & 2.551 & 1.707 & 10.064 \\
\hline 5. Baixo-Superior & 674 & 1.697 & 2.287 & 2.317 & 4.867 & 3.008 & 14.848 \\
\hline 6. Baixo-Inferior & 475 & 1.659 & 2.197 & 3.313 & 6.200 & 9.408 & 23.252 \\
\hline TOTAL DOS INDIVÍDUOS & \multirow{2}{*}{3.853} & \multirow{2}{*}{7.504} & \multirow{2}{*}{8.353} & \multirow{2}{*}{9.037} & \multirow{2}{*}{15.721} & \multirow{2}{*}{15.205} & \multirow{2}{*}{59.673} \\
\hline$N=59.673$ & & & & & & & \\
\hline
\end{tabular}

Fonte: Elaborado pelos autores a partir dos microdados da PNAD de 2014 (IBGE). 
A partir da Tabela 1 podem ser calculadas várias medidas de mobilidade, conforme Yasuda (1964). Considerando o número total de pessoas da amostra, $\quad N=\sum_{i} \sum_{j} n_{i j}$, a mobilidade total (\%) será definida como a diferença entre $\mathrm{N}$ e os indivíduos imóveis, ou seja, aqueles que estão na diagonal principal, $\sum_{i=1}^{k} n_{i i}$ dividida pelo total da amostra $(N)$ :

Mobilidade Total (\%): $\frac{1}{N}\left(N-\sum_{i=1}^{k} n_{i i}\right) 100$

A seguir podemos calcular as mobilidades estrutural e circular (\%) por meio das equações (4) e (5), onde $\bar{n}_{i i}$ é o mínimo entre o total da linha $\left(n_{i \bullet}\right)$ e o total da coluna $\left(n_{\bullet}\right)$ correspondente.

Mobilidade Estrutural (\%): $\frac{1}{N}\left(N-\sum_{i=1}^{k} \bar{n}_{i i}\right) 100$

Mobilidade Circular (\%): $\frac{1}{N}\left(\sum_{i=1}^{k} \bar{n}_{i i}-\sum_{i=1}^{k} n_{i i}\right) 100$

No caso hipotético de uma matriz com valores não nulos apenas na diagonal, teríamos $n_{i i}=n_{i \bullet}=n_{\bullet i}=\bar{n}_{i i}$ e tanto a mobilidade estrutural como a mobilidade circular seriam nulas. A diferença $N-\sum_{i=1}^{k} \bar{n}_{i i}$ é o número de pessoas que necessariamente está fora da diagonal devido ao fato de totais de coluna serem diferentes dos respectivos totais de linha, isto é, o número de pessoas que necessariamente apresenta mobilidade porque a distribuição entre os estratos no destino é diferente da distribuição na origem. É nesse sentido que é considerada mobilidade "estrutural". 
Yasuda (1964) nos oferece outro índice: o Coeficiente de Abertura (Y). Ele avalia o grau de flexibilidade da estrutura social através da aproximação de uma situação em que as distribuições de origem e destino são independentes. "Um coeficiente $Y$ com valor 1 nem sempre significa, teoricamente, uma sociedade com mobilidade perfeita, porém uma sociedade com mobilidade perfeita sempre terá $Y$ igual a 1. (...), um coeficiente $Y$ igual a 1 quase sempre significará perfeita mobilidade" (Yasuda, 1964, p. 18).

$$
Y=\frac{\sum_{i=1}^{k} \bar{n}_{i i}-\sum_{i=1}^{k} n_{i i}}{\sum_{i=1}^{k} \bar{n}_{i i}-\sum_{i=1}^{k} \frac{n_{i \bullet} \times n_{\bullet i}}{N}}
$$

Por fim, propomos a utilização do status socio-ocupacional na construção de uma variável que expresse uma magnitude da mobilidade experimentada pelos indivíduos nos dois cenários: intergeracional e intrageracional. A nova variável é construída como a diferença dos logaritmos do SSE da ocupação de destino e do SSE da ocupação de origem.

Para o caso intergeracional, considera-se o logaritmo do SSE da ocupação do filho e o logaritmo do SSE da ocupação do pai quando o filho tinha 15 anos; no intrageracional utiliza-se o logaritmo do SSE da ocupação atual e o logaritmo do SSE da primeira ocupação. A utilização dos escores de SSE em logaritmo justifica-se pelo método empregado na construção do SSE, que é essencialmente uma variável de rendimento.

O interesse da análise é identificar se há evidência, na sociedade brasileira, da relação entre o grau de mobilidade e características individuais como migração e cor. Com esse propósito, estimamos equações de regressão múltipla utilizando o Método dos Mínimos Quadrados Ponderados.

Para esse exercício, foram mantidos na amostra os indivíduos pretos, pardos e brancos e excluídos amarelos, indígenas e aqueles sem declaração de cor. Após a depuração, a amostra ficou com 22.762 indivíduos, representando uma população de 59,2 milhões dos quais 58,9\% são 
homens, 41,1\% mulheres, 49,5\% são brancos, 41,7\% pardos, 8,8\% pretos e $28,1 \%$ são considerados migrantes, isto é, pessoas que não residem na Unidade da Federação (UF) em que nasceram ou que já tenham morado em outra UF ou fora do país.

Inicialmente são propostos os modelos (7) e (8), a seguir, para verificar efeitos das variáveis migração e cor sobre o grau de mobilidade:

$$
\begin{aligned}
& D L S S E=\alpha+\beta_{1} M+u \\
& D L S S E=\alpha+\beta_{1} M+\beta_{2} N+\beta_{3} M N+u
\end{aligned}
$$

Nos dois modelos, DLSSE é a variável diferença dos logaritmos do status socio-ocupacional de destino e de origem; $M$, é uma variável binária que assume valor 1 para indivíduos migrantes e 0 em caso contrário. A variável $N$ é uma binária que assume valor 1 para os indivíduos negros (pretos e pardos) e 0 para os brancos. MN é a variável de interação entre migração $(M)$ e cor $(N)$.

Para testar a robustez das relações entre mobilidade, migração e cor, também foram ajustados outros dois modelos, com a adição de variáveis de controle:

$$
\begin{gathered}
\text { DLSSE }=\alpha+\beta M+\sum_{j} \delta_{j} E_{j}+\sum_{h} \gamma_{h} I_{h}+\sum_{v} \theta_{v} R_{v}+u_{i} \\
D L S S E=\alpha+\beta_{1} M+\beta_{2} N+\beta_{3} M N+\sum_{j} \delta_{j} E_{j}+\sum_{h} \gamma_{h} I_{h}+\sum_{v} \theta_{v} R_{v}+u_{i}
\end{gathered}
$$

As variáveis de controle em (9) e (10) são: $E$, variável que engloba 15 variáveis binárias $\left(E_{j}, \operatorname{com} j=1, \ldots, 15\right)$ para distinguir 16 níveis de escolaridade, sendo $E_{j}=1$ para a $j$-ésima faixa e $E_{j}=0$ nas demais, sendo a base formada 
por indivíduos sem escolaridade; 9 variáveis binárias $\left(I_{h^{\prime}} \operatorname{com} h=1, \ldots, 9\right)$ para distinguir as 10 faixas de idade (16 a 20 anos, 21 a 25, ..., 61 a 65), fazendo $I_{h}=1$ na $h$-ésima faixa e $I_{h}=0$ nas demais; $R_{v}$ representa cinco variáveis binárias para a região de residência (Norte, Centro-Oeste, Sul, Sudeste - exceto SP, e São Paulo), sendo a região Nordeste a referência.

Não consideramos, neste trabalho, a metodologia de análise da mobilidade fundamentada em um modelo de regressão linear do logaritmo do rendimento do filho contra o do pai. $^{2} \mathrm{O}$ respectivo coeficiente de regressão $\left(b_{x}\right)$ é uma estimativa da elasticidade do rendimento do filho em relação ao rendimento do pai e $1-b_{x}$ indica o grau de mobilidade. Cabe ressaltar que o valor desse coeficiente de regressão seria igual a 1, indicando absoluta imobilidade, se o rendimento dos filhos fosse, por exemplo, sempre igual ao dobro do rendimento dos respectivos pais. Isso mostra que, nessa metodologia, o valor de $1-b_{x}$ mede apenas a mobilidade relativa, sendo insensível à mobilidade estrutural.

\section{Resultados e discussão}

A estrutura social obtida divide as ocupações em seis estratos: Alto, Médio-Superior, Médio-Médio, Médio-Inferior, Baixo-Superior e BaixoInferior. O intervalo de cada estrato é definido como maior ou igual ao limite inferior e menor que o limite superior.

A tabela 2, a seguir, mostra os limites de cada estrato e a distribuição da população de 2014 entre eles. Nota-se uma grande participação nos estratos baixos, onde estão cerca de $54,2 \%$ dos indivíduos. O restante da população está alocado nos estratos médios e alto, 40,1\% e 5,7\%, respectivamente.

A matriz de mobilidade apresentada na tabela 3 diz respeito à mobilidade intergeracional, que abrange um intervalo de aproximadamente 50 anos (1965-2014); as porcentagens nas seis primeiras colunas de

\footnotetext{
${ }^{2}$ Ver, por exemplo, Ferreira e Veloso (2006).
} 
números mostram, para cada estrato socio-ocupacional paterno, como os filhos se distribuem pelos seis estratos socio-ocupacionais. A primeira linha, por exemplo, nos diz que os filhos de pais com ocupações de status alto estão alocados da seguinte maneira: 37,6\% continuam no estrato alto, 26,4\% estão no estrato médio-superior, 15,3\% no médio-médio e assim por diante, até o estrato baixo-inferior, onde estão 2,8\%. Essas informações indicam que $62,4 \%$ dos filhos de pais em estrato alto experimentaram mobilidade descendente.

Tabela 2 - Limites dos estratos socio-ocupacionais e distribuição da população para o Brasil em 2014

\begin{tabular}{l|c|c|c|c|c}
\hline \multirow{2}{*}{$\begin{array}{c}\text { Estrato } \\
\text { Ocupacional }\end{array}$} & \multicolumn{2}{|c|}{ Limites } & \multicolumn{2}{c}{ Distribuição da população } \\
\cline { 2 - 6 } & $\begin{array}{c}\text { Limite } \\
\text { Inferior }\end{array}$ & $\begin{array}{c}\text { Limite } \\
\text { Superior }\end{array}$ & $\begin{array}{c}\text { Pessoas } \\
(1000)\end{array}$ & Porcentagem \% & $\begin{array}{c}\text { Porcentagem } \\
\text { cumulativa \% }\end{array}$ \\
\hline Alto & 28,97 & 100 & 4.886 & 5,71 & 5,71 \\
\hline Médio-Superior & 19,79 & 28,97 & 10.160 & 11,86 & 17,57 \\
\hline Médio-Médio & 13,68 & 19,79 & 11.178 & 13,05 & 30,62 \\
\hline Médio-Inferior & 10,96 & 13,68 & 12.974 & 15,15 & 45,77 \\
\hline Baixo-Superior & 9,36 & 10,96 & 23.646 & 27,61 & 73,38 \\
\hline Baixo-Inferior & 0 & 9,36 & 22.798 & 26,62 & 100 \\
\hline
\end{tabular}

Fonte: Elaborado pelos autores a partir dos microdados da PNAD de 2014 (IBGE).

Houve, porém, um aumento da presença dos filhos no estrato alto, como pode ser verificado nos totais. A participação nesse estrato é três pontos percentuais (p.p.) maior que na distribuição dos pais. Nos estratos médios esse fenômeno é mais acentuado: enquanto apenas 32,6\% dos pais compõem os estratos médios, 41,7\% dos filhos alcançaram ocupações nesses estratos. Essa melhora no status das ocupações dos filhos em relação aos seus pais deve-se, principalmente, à redução da participação nos estratos baixos que, para os pais, era $63,9 \%$ e para os filhos caiu para $51,8 \%$. 
Tabela 3 - Matriz de mobilidade intergeracional brasileira para 2014

\begin{tabular}{|c|c|c|c|c|c|c|c|c|}
\hline \multirow[b]{2}{*}{ STATUS DO PAI } & \multicolumn{6}{|c|}{ STATUS DO FILHO EM 2014} & \multirow[b]{2}{*}{ TOTAL } & \multirow{2}{*}{$\begin{array}{c}\text { TOTAL } \\
\text { DOS } \\
\text { PAIS }\end{array}$} \\
\hline & 1 & 2 & 3 & 4 & 5 & 6 & & \\
\hline 1. Alto & 37,6 & 26,4 & 15,3 & 5,5 & 12,4 & 2,8 & 100,0 & 3,5 \\
\hline 2. Médio-Superior & 17,9 & 28,2 & 17,6 & 10,7 & 17,1 & 8,3 & 100,0 & 7,4 \\
\hline 3. Médio-Médio & 9,9 & 19,4 & 21,0 & 14,8 & 21,8 & 13,1 & 100,0 & 8,3 \\
\hline 4. Médio-Inferior & 6,2 & 13,7 & 17,1 & 20,7 & 25,4 & 17,0 & 100,0 & 16,9 \\
\hline 5. Baixo-Superior & 4,5 & 11,4 & 15,4 & 15,6 & 32,8 & 20,3 & 100,0 & 24,9 \\
\hline 6. Baixo-Inferior & 2,0 & 7,1 & 9,4 & 14,2 & 26,7 & 40,5 & 100,0 & 39,0 \\
\hline TOTAL DOS INDIVIIDUOS & \multirow[b]{2}{*}{6,5} & \multirow[b]{2}{*}{12,6} & \multirow[b]{2}{*}{14,0} & \multirow[b]{2}{*}{15,1} & \multirow[b]{2}{*}{26,3} & \multirow[b]{2}{*}{25,5} & \multirow[b]{2}{*}{100,0} & \multirow[b]{2}{*}{100,0} \\
\hline $\mathrm{N}=59.673$ milhares & & & & & & & & \\
\hline
\end{tabular}

Fonte: Elaborado pelos autores a partir dos microdados da PNAD de 2014 (IBGE).

Outra característica das matrizes de mobilidade é a possibilidade de se analisar a imobilidade e as mobilidades ascendente e descendente. Na diagonal principal são representados os indivíduos considerados imóveis, ou seja, que estão nos mesmos estratos socio-ocupacionais dos seus pais. As entradas acima da diagonal principal caracterizam os indivíduos que experimentaram mobilidade descendente e, abaixo dela, os que tiveram mobilidade ascendente.

A tabela 4 sumariza as características gerais da mobilidade socioocupacional intergeracional brasileira. É possível afirmar que a parcela dos indivíduos móveis (67\%), daqueles que melhoraram ou pioraram, supera com folga a dos imóveis (33\%). Dentre os indivíduos móveis, predomina o movimento ascendente, ou seja, ainda que não seja desprezível a quantidade de indivíduos que experimentaram uma piora de status, a maioria se encontra em ocupações de status superior à dos pais. 
Quando analisamos as medidas de mobilidade estrutural e mobilidade circular, constatamos que o número de indivíduos que subiu na estrutura social devido à criação de novas oportunidades (estrutural) é inferior aos que subiram devido à troca de posições e aposentadorias (circular). O resultado do Coeficiente de Abertura (Y), ou Índice de Yasuda, de 0,82, é reflexo da alta mobilidade circular e evidencia que a estrutura socio-ocupacional brasileira no período é marcada por alta fluidez e mobilidade.

Tabela 4 - Características da mobilidade intergeracional brasileira para 2014

\begin{tabular}{l|l}
\hline Medida & Valor \\
\hline Mobilidade Ascendente (\%) & 44,9 \\
Imobilidade (\%) & 32,6 \\
Mobilidade Descendente (\%) & 22,5 \\
Mobilidade Total (\%) & 67,4 \\
Mobilidade Estrutural (\%) & 15,2 \\
Mobilidade Circular (\%) & 52,2 \\
Coeficiente de Abertura (Y) & 0,823 \\
\hline
\end{tabular}

Fonte: Elaborado pelos autores a partir dos microdados da PNAD de 2014 (IBGE).

Partindo para a análise intrageracional, na qual verificamos a mobilidade de carreira dos indivíduos até 2014, podemos observar novamente substancial mobilidade. A tabela 5 apresenta a matriz de mobilidade intrageracional para 2014.

A primeira observação a ser feita é o início de carreira em ocupações de status predominantemente baixo, cerca de 76,9\% dos indivíduos (soma dos estratos Baixo-Inferior e Baixo-Superior, 51,0 + 25,9 = 76,9). A tabela 5 mostra que os movimentos de queda para os estratos baixos são mais fracos que os movimentos de ascensão a partir desses estratos, de maneira 
que, no final da sua carreira, 51,8\% $(26,3+25,5=51,8)$ das pessoas estão nesses estratos.

Tabela 5 - Matriz de mobilidade intrageracional brasileira para 2014

\begin{tabular}{|c|c|c|c|c|c|c|c|c|}
\hline \multirow{2}{*}{$\begin{array}{c}\text { STATUS DA PRIMEIRA } \\
\text { OCUPAÇÃO }\end{array}$} & \multicolumn{6}{|c|}{ STATUS DO INDIVÍDUO EM 2014} & \multirow{2}{*}{ TOTAL } & \multirow{2}{*}{$\begin{array}{l}\text { TOTAL DO } \\
\text { STATUS } \\
\text { INICIAL }\end{array}$} \\
\hline & 1 & 2 & 3 & 4 & 5 & 6 & & \\
\hline 1. Alto & 75,0 & 13,7 & 6,9 & 1,9 & 1,6 & 1,0 & 100,0 & 1,9 \\
\hline 2. Médio-Superior & 15,9 & 55,7 & 13,6 & 5,3 & 7,3 & 2,2 & 100,0 & 4,3 \\
\hline 3. Médio-Médio & 13,3 & 21,5 & 39,7 & 7,8 & 12,6 & 5,2 & 100,0 & 10,0 \\
\hline 4. Médio-Inferior & 3,9 & 13,3 & 12,0 & 43,8 & 18,9 & 8,0 & 100,0 & 6,9 \\
\hline 5. Baixo-Superior & 6,7 & 14,3 & 14,6 & 14,6 & 38,8 & 11,0 & 100,0 & 25,9 \\
\hline 6. Baixo-Inferior & 2,0 & 6,2 & 9,2 & 14,3 & 26,3 & 42,1 & 100,0 & 51,0 \\
\hline $\begin{array}{l}\text { TOTAL DOS } \\
\text { INDIVÍDUOS } \\
\mathrm{N}=59.673 \text { milhares }\end{array}$ & 6,5 & 12,6 & 14,0 & 15,1 & 26,3 & 25,5 & 100,0 & 100,0 \\
\hline
\end{tabular}

Fonte: Elaborado pelos autores a partir dos microdados da PNAD de 2014 (IBGE).

Os números da diagonal principal indicam que há alta imobilidade na carreira dos brasileiros, principalmente nos extremos da estrutura. Em 2014, nada menos que 75,0\% dos indivíduos que iniciaram suas carreiras no estrato alto permaneciam nesse mesmo estrato. No estrato baixo-inferior a permanência (imobilidade) é de 42,1\%.

A participação em ocupações do estrato alto, em 2014, era aproximadamente três vezes maior do que no início da carreira dos indivíduos. É possível concluir que houve uma melhora significativa nas ocupações dos indivíduos ao longo de suas carreiras. A análise da mobilidade intrageracional brasileira mostra grande imobilidade e também grande número de indivíduos que ascenderam a ocupações melhores; apenas 9,0\% experimentaram mobilidade descendente. A imobilidade elevada 
se manifesta, principalmente, na grande proporção de indivíduos que iniciaram suas carreiras em ocupações de elevado status socioeconômico e se mantiveram nelas. Para os demais indivíduos é substancial a chance de se alocar em ocupações melhores ao longo de suas carreiras.

As medidas de mobilidade apresentadas na tabela 6 mostram um comportamento diferente da mobilidade intergeracional. A diferença entre a mobilidade circular e a estrutural é menor, 6,7 p.p., do que para a análise intergeracional onde a diferença entre as duas é de 37,0 p.p. Isso demonstra que um maior número de indivíduos experimentou mobilidade graças à criação de novas oportunidades no mercado de trabalho. Uma vez que o coeficiente de abertura ( $Y$ ) é sensível à grandeza da mobilidade circular, é observada uma menor fluidez na mobilidade intrageracional, quando comparada com a intergeracional.

Tabela 6 - Características da mobilidade intrageracional brasileira para 2014

\begin{tabular}{l|c}
\hline Medida & 2014 \\
\hline Mobilidade Ascendente (\%) & 48,7 \\
Imobilidade (\%) & 42,3 \\
Mobilidade Descendente (\%) & 9,0 \\
Mobilidade Total (\%) & 57,7 \\
Mobilidade Estrutural (\%) & 25,5 \\
Mobilidade Circular (\%) & 32,2 \\
Coeficiente de Abertura (Y) & 0,625 \\
\hline
\end{tabular}

Fonte: Elaborado pelos autores a partir dos microdados da PNAD de 2014 (IBGE).

É oportuno apresentar uma breve comparação com os resultados obtidos em trabalhos anteriores. Ainda que os resultados não sejam rigorosamente comparáveis devido a mudanças metodológicas na construção dos estratos socio-ocupacionais e nos critérios de obtenção das amostras, é importante 
contextualizar o cenário obtido para 2014 com os do período anterior, 1996. Os estudos comparados de Pastore e Silva (2000) e Oliveira (2018) também fazem uso de escalas socio-ocupacionais, matrizes de transição de status e medidas de mobilidade, e nos fornecem uma visão da mobilidade brasileira para o ano de 1996 a partir dos dados fornecidos pelo IBGE no Suplemento de Mobilidade Socio-ocupacional da PNAD/1996.

Para a mobilidade intergeracional, em 1996, Pastore e Silva (2000) estimaram a mobilidade social total em $63,2 \%$, com $49,6 \%$ de movimentos ascendentes e 13,6\% descendentes. Aplicando-se aos dados da PNAD de 1996 o procedimento metodológico apresentado aqui, como em Oliveira (2018), verifica-se que a mobilidade social é de $65,8 \%$, com 49,5\% de mobilidade ascendente e $16,3 \%$ descendente. São resultados muito próximos, com diferenças certamente devidas às alterações na metodologia adotada. Comparando-se com os resultados de 2014, observamos certa estabilidade nos padrões de mobilidade intergeracional, mas é importante sinalizar que houve aumento na proporção de movimentos descendentes e redução na porcentagem de movimentos ascendentes em 2014.

Pastore e Silva (2000) obtêm medidas de mobilidade circular e estrutural, para 1996, de aproximadamente 31\% cada, com a primeira superando a segunda em apenas 0,4 p.p. Por outro lado, Oliveira (2018) estima a mobilidade circular em 38\%, superando a estrutural em 10 p.p. Como a metodologia desse segundo trabalho é semelhante à utilizada neste estudo, ele será adotado como base de comparação. Em 2014 ocorre um distanciamento ainda maior entre mobilidade circular e estrutural, com a primeira aumentando em detrimento da segunda, chegando a 52\%, 37 p.p. acima da mobilidade estrutural. Esse resultado explica o aumento do Coeficiente de Abertura (Y) entre os dois períodos, de 0,76 em 1996, segundo Oliveira (2018), para 0,82 em 2014.

A mobilidade intrageracional apresenta diferenças mais significativas entre os estudos de Pastore e Silva (2000) e Oliveira (2018). A mudança intrageracional mostra-se mais sensível à estrutura socio-ocupacional utilizada 
na análise. Em 1996, 61\% dos indivíduos eram, de acordo com Pastore e Silva (1996), imóveis, mas, com base no procedimento utilizado aqui, esse número é de aproximadamente $42 \%$. A porcentagem de indivíduos com mobilidade descendente nos dois estudos é próxima, sendo 9\% no primeiro estudo e 13\% no segundo. A diferença maior, portanto, está na proporção de indivíduos com mobilidade ascendente na dinâmica intrageracional: $30 \%$ em Pastore e Silva e mais de 45\% com a nova metodologia.

Utilizando o mesmo procedimento metodológico, verifica-se que a mobilidade intrageracional ascendente passa de 45\% em 1996 para 49\% em 2014. Apesar da aparente melhora nas oportunidades de carreira, ao comparar os resultados do Coeficiente de Abertura $(\mathrm{Y})$ entre os dois períodos, 0,66 em 1996 e 0,62 em 2014, há indicação de que houve aumento na rigidez do mercado de trabalho. Esse resultado é consequência da diminuição da diferença entre a mobilidade circular e estrutural nos dois períodos. Tanto em 1996 quanto em 2014 a mobilidade circular supera a estrutural, mas a distância entre as duas diminuiu de 17 p.p. para 10 p.p.

A tabela 7 apresenta os resultados dos modelos estimados para a análise da influência da migração e da cor do indivíduo sobre a mobilidade socio-ocupacional experimentada. Os modelos estão denominados de acordo com suas equações correspondentes na seção metodológica deste trabalho e considerando a mobilidade intergeracional ou a intrageracional. Todos os quatro modelos foram estimados utilizando dados de 22.762 observações, como explicado anteriormente. A tabela não mostra os coeficientes de determinação $\left(R^{2}\right)$, que são sempre inferiores a 1,2\%. Ainda que tais regressões sejam inapropriadas para prever a mobilidade socioocupacional individual, todos os testes $\mathrm{F}$ são significativos ao nível de 1\%, mostrando que as equações captam a existência de relação entre a magnitude da mobilidade e as variáveis explanatórias incluídas nos modelos, o que permite rejeitar a hipótese de que os coeficientes de regressão são nulos.

Como a variável dependente se refere ao logaritmo do status socioeconômico (SSE), a interpretação dos resultados é facilitada calculando- 
se a variação percentual no valor esperado do SSE associada a uma mudança de 0 para 1 em uma variável explanatória, que chamamos de efeito dessa variável. Sendo $\hat{\beta}$ a estimativa do coeficiente de regressão, o respectivo efeito é

$$
\text { Efeito }=100[\exp (\hat{\beta})-1]
$$

Para a primeira equação de regressão apresentada na Tabela 7 , a estimativa do coeficiente de regressão é 0,0249. Isso significa, nessa regressão, que um migrante apresenta mobilidade socio-ocupacional esperada $100[\exp (0,0249)-1]=2,52 \%$ maior do que um não migrante.

Os resultados apresentados na Tabela 7 consistem nos modelos estimados utilizando exclusivamente as variáveis de interesse: magnitude da mobilidade, migração e cor. Esses modelos visam identificar a influência dessas duas últimas na primeira. Analisamos primeiro o impacto dessas na mobilidade intergeracional, e depois na mobilidade intrageracional.

Quando estimamos a relação entre a magnitude da mobilidade intergeracional do indivíduo e sua característica de migrante, modelo 7, observamos um efeito positivo e significativo a 1\%; os migrantes experimentam uma mobilidade socio-ocupacional 2,5\% maior que os não migrantes. Quando adicionamos a cor do indivíduo, modelo 8, a estimativa do coeficiente de $M$ só é estatisticamente diferente de zero ao nível de significância de 10\%, mas cabe ressaltar que o teste $\mathrm{F}$ para a hipótese de nulidade simultânea dos coeficientes de $M$ e de $M N$ é significativo a 1\%. A variável de interação entre migração e cor apresenta um resultado positivo, indicando que o efeito positivo de ser migrante é maior para indivíduos negros $(3,3 \%=100[\exp (0,0191+0,0135)])$ do que para brancos $(1,9 \%)$, ainda que o coeficiente da interação não seja, isoladamente, estatisticamente significativo. A interação também faz com que o efeito de ser negro seja menos intenso para os migrantes $(-3,0 \%=100[\exp (0,0443+0,0135)])$ do que para os não migrantes $(-4,3 \%)$. 


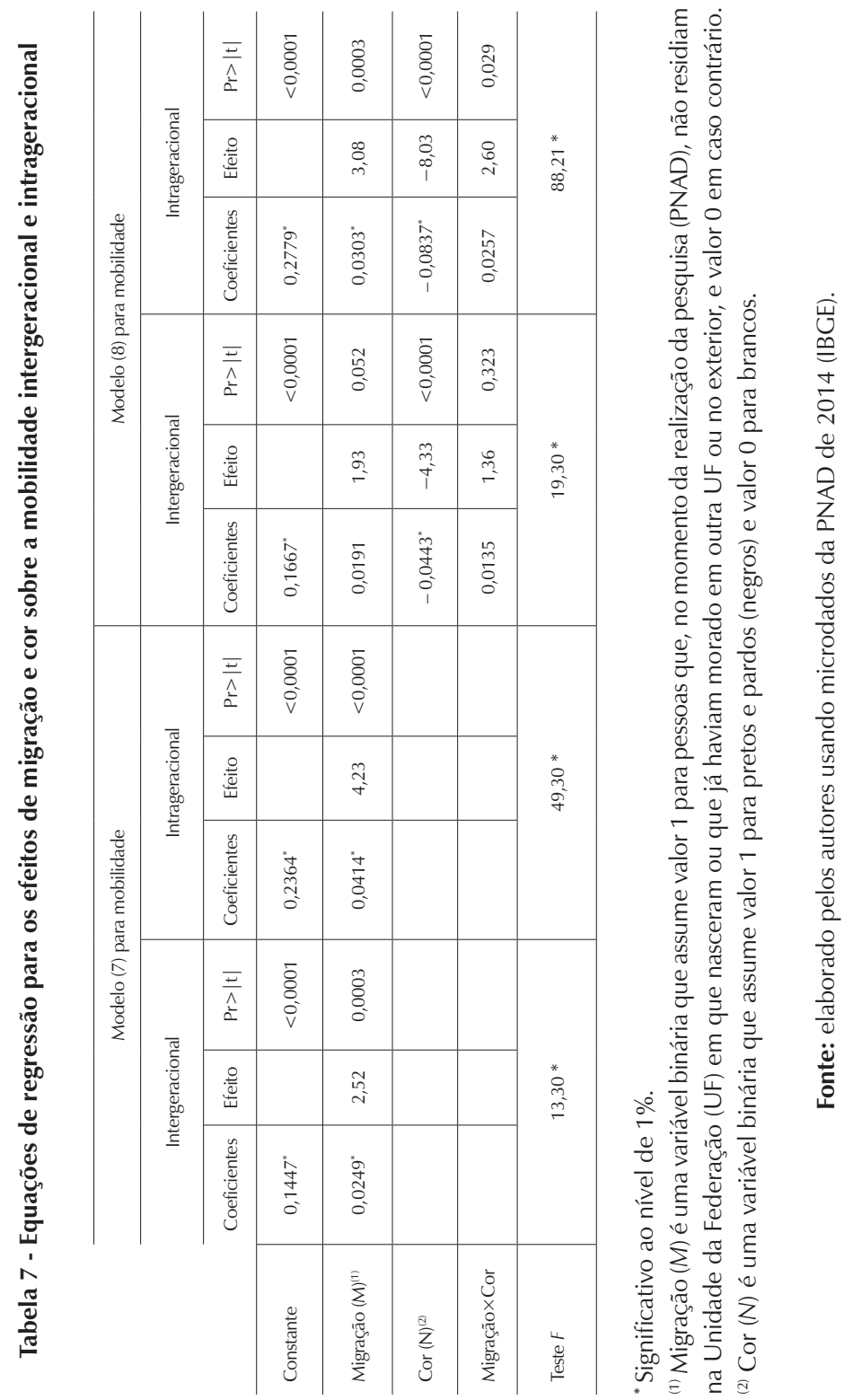

Sociologias, Porto Alegre, ano 23, n. 57, mai-ago 2021, p. 208-238. 
No caso da mobilidade intrageracional as relações mantêm o mesmo sentido. No modelo que analisa apenas o efeito de ser migrante, esse é estimado como um acréscimo de 4,2\% no SSE esperado. Quando adicionamos cor e sua interação com migração, modelo 8, verifica-se que o coeficiente da interação entre cor e migração se mantém positivo, como no caso da mobilidade intergeracional, mas passa a ser significativo a $5 \%$. Os coeficientes indicam que o fato de ser migrante faz com que o SSE esperado de um branco seja 3,1\% maior, enquanto para um negro esse efeito é 5,8\%. O efeito de ser negro para um migrante (-5,6\%) é menos intenso que o efeito de ser negro para pessoa não migrante $(-8,0 \%)$.

Quando estimamos modelos incluindo diversas variáveis de controle, como descrito anteriormente, o valor estimado do efeito de ser migrante se torna menor, já que tanto a mobilidade social como a probabilidade de ser migrante estão associadas à escolaridade, à idade e à região de residência. Verifica-se, entretanto, que o efeito de ser migrante permanece positivo e maior para os negros do que para os brancos. ${ }^{3}$ Tais resultados corroboram os obtidos por Jesus (2020) para o rendimento das pessoas.

\section{Conclusões}

A nova metodologia de estratificação proposta neste trabalho contribui com o avanço teórico do estudo de mobilidade socio-ocupacional ao oferecer uma hierarquização das ocupações presentes na CBO-Domiciliar, sem as arbitrariedades encontradas em métodos anteriores e baseada nas especificidades da sociedade brasileira.

A mobilidade socio-ocupacional brasileira apresenta dinamismo e fluidez. Os resultados mostram que, como sugere a literatura, a mobilidade descendente é inferior à ascendente e à imobilidade, mas não é desprezível. Analisando as matrizes de mobilidade para os casos intergeracional e intrageracional, nota-se que a estrutura social brasileira experimentou um esvaziamento dos estratos baixos em favor dos estratos médios.

${ }^{3}$ Os resultados completos obtidos com os diferentes modelos podem ser fornecidos aos interessados (cahliveira42@gmail.com). 
Em 2014, 44,9\% dos indivíduos estava em ocupações de status socioeconômico superior à de seus pais, enquanto 32,6\% permaneceu em ocupações de status similar e $22,5 \%$ de status inferior. Ao comparar as diversas formas de mobilidade intergeracional, verifica-se que a mobilidade circular é a mais expressiva, elevando o coeficiente de abertura (Y). No Brasil, a movimentação dos indivíduos entre os estratos socio-ocupacionais aconteceu em maior medida devido a substituições nos postos de trabalho já disponíveis no mercado (mobilidade circular). A mobilidade associada com a abertura de novas oportunidades no mercado de trabalho (mobilidade estrutural) também foi substancial, mas menor do que a circular.

Quando analisamos a mobilidade intrageracional, a redução da presença em ocupações de baixo status é mais expressiva. Os dados da PNAD de 2014 mostram que 76,9\% dos indivíduos iniciaram suas carreiras em ocupações dos estratos baixos e que essa participação foi reduzida em 25 p.p. na ocupação declarada no ano da pesquisa.

Concluímos que não se concretizaram as previsões de diminuição da mobilidade social brasileira após a década de 1990 (Albuquerque; Velloso, 1993; Pastore; Haller, 1993) e que a tendência de crescimento da mobilidade apontada pelos trabalhos de Scalon (1999), Pastore e Silva (2000) e Ribeiro (2007) se manteve. No entanto, a mobilidade estrutural, ao contrário do que se esperava levando em conta as transformações pelas quais passou o mercado de trabalho brasileiro no período, foi inferior à mobilidade circular. Assim, os indivíduos que ascenderam ou descenderam na estrutura socio-ocupacional o fizeram majoritariamente por meio da substituição e das trocas entre postos de trabalho já existentes, bem como percorreram curtas distâncias, indo para estratos adjacentes aos de origem.

A análise da influência de variáveis como migração e cor na magnitude da mobilidade socio-ocupacional experimentada nos dois cenários, intergeracional e intrageracional, mostra que os negros (pretos e pardos) enfrentam maior dificuldade de ascensão ocupacional, que os indivíduos migrantes apresentam maior mobilidade do que os não migrantes e que esse efeito positivo associado à migração é maior para os negros. 
O estudo dos movimentos dos indivíduos na estrutura socio-ocupacional brasileira proporciona um panorama das oportunidades às quais eles estão expostos. Ainda que não haja na literatura um consenso sobre a melhor metodologia a ser empregada neste tipo de análise, as matrizes de mobilidade oferecem uma abordagem didática e ilustrativa. Os resultados obtidos neste trabalho mostram que essa metodologia oferece um diagnóstico muito próximo ao de outros estudos que constataram uma elevada mobilidade total e ascendente para a sociedade brasileira a partir da utilização dos dados da PNAD de 2014 (Ribeiro, 2017; Jannuzzi; Montagner; Taira, 2019).

Há, certamente, um longo caminho a ser percorrido para que se estabeleça uma metodologia de análise da mobilidade social de ampla aceitação, que permita a comparação dos resultados entre sociedades e períodos distintos.

Camilla Oliveira é doutoranda em Economia Aplicada na Escola Superior de Agricultura Luiz de Queiroz - ESALQ/USP. $₫$ cahliveira42@gmail.com

Rodolfo Hoffmann é Doutor em Economia Agrária e Professor Titular na Escola Superior de Agricultura Luiz de Queiroz - ESALQ/USP. $₫ \underline{\text { hoffmannr@usp.br }}$

\section{Referências}

1. ABRAMITZKY, Ran; BOUSTAN, Leah P.; JÁCOME, Elisa; PÉREZ, Santiago. Intergenerational mobility of immigrants in the US over two centuries. National Bureau of Economic Research, working paper n. 26408, out. 2019. https://doi. org/10.3386/w26408

2. AGHEVLI, Bijan B.; MEHRAN, Farhad. Optimal grouping of income distribution data. Journal of the American Statistical Association, v. 76, n. 373, p. 22-26, 1981. http://doi.org/10.1080/01621459.1981.10477596

3. AlBuQueRQuE, Roberto C. D.; VEllOSO, João Paulo D. R. Pobreza e mobilidade social. Pobreza e mobilidade social: as bases do desenvolvimento moderno. São Paulo: Nobel, p. 15-21, 1993.

4. BLAU, Peter M.; DUNCAN, Otis D. The American occupational structure. Nova York: The Free Press, 1967. 
5. BREEN, Richard. Individual level models for mobility tables and other crossclassifications. Sociological Methods and Research, v. 23, p. 147-173. 1994. https://doi.org/10.1177\%2F0049124194023002001

6. DAVIES, James B.; SHORROCKS, Anthony F. Optimal grouping of income and wealth data. Journal of Econometrics, v. 42, n. 1, p. 97-108, 1989. https://doi. org/10.1016/0304-4076(89)90078-X

7. DAVIS, Kingsley. Human society. Nova York: Macmillan, 1949.

8. DAVIS, Kingsley; MOORE, Wilbert E. Some principles of stratification. American Sociological Review, v. 10, n. 2, p. 242-249, 1945. https://doi.org/10.2307/2085643

9. DIPRETE, Thomas. Adding covariates to loglinear models for the studies of social mobility. American Sociological Review, v. 55, p. 757-773, 1990. https://doi.org/10.2307/2095870

10. ERIKSON, Robert; GOLDTHORPE, John H.; PORTOCARERO, Lucienne. Intergenerational class mobility in three Western European societies: England, France and Sweden. British Journal of Sociology, v. 30, n. 4, p. 415-441, 1979. https://doi.org/10.2307/589632

11. FERREIRA, Sergio G.; VELOSO, Fernando A. Intergenerational mobility of wages in Brazil. Brazilian Review of Econometrics, v. 26, n. 2, p. 181-211, 2006. https://doi.org/10.12660/bre.v26n22006.1576

12. GLASS, David V.; HALL, John R. Social mobility in Great Britain: A study in intergenerational change in status. In: GLASS, David V. Social Mobility in Great Britain. Londres: Routledge and Kegan Paul, 1954.

13. GOLDTHORPE, John H.; HALSEY, Albert H.; HEATH, Anthony; RIDGE, John; BLOOM, Leonard; JONES, Frank L. Social mobility and class structure in modern Britain. Oxford: Claredon Press, 1982.

14. GOODMAN, Leo A. Multiplicative models for the analysis of occupational mobility tables and other kinds of cross-classification tables. American Journal of Sociology, v. 84, n. 4, p. 804-819, 1979. https://doi.org/10.1086/226862

15. HAYES, Bernadette C.; MILLER, Robert L. The silenced voice: female social mobility patterns with particular reference to the British Isles. British Journal of Sociology, v. 44, n. 4, p. 653-672, 1993.

16. HENDRICKX, John; GANZEBOOM, Harry B. G. Occupational status attainment in the Netherlands, 1920-1990: A multinomial logistic analysis. European Sociological Review, v. 14, p. 387-403, 1998. https://doi.org/10.1093/ oxfordjournals.esr.a018246

17. HERTEL, Florian R. Social mobility in the 20th Century: Class mobility and occupational change in the United States and Germany. Wiesbaden: Springer VS, 2017.

18. HOFFMANN, Rodolfo. Desigualdade de renda no Brasil em 2005 e sua decomposição. Centro de Gestão e Estudos Estratégicos. II Seminário de Análise dos Resultados da Pesquisa Nacional por Amostra de Domicílios, 2005a. 
19. HOFFMANN, Rodolfo; SIMÃO, Rosycler C. S. Determinantes do rendimento das pessoas ocupadas em Minas Gerais em 2000: o limiar no efeito da escolaridade e as diferenças entre mesorregiões. Nova Economia, 15, n. 2, p. 35-62, 2005b.

20. HOUT, Michael. Mobility tables. Beverly Hills: Sage Publications, 1983.

21. HUTCHINSON, Bertram A. Mobilidade e trabalho: um estudo na cidade de São Paulo. Brasília: Centro Brasileiro de Pesquisas Educacionais, INEP, MEC, 1960.

22. JANNUZZI, Paulo M. Migração e mobilidade social: migrantes no mercado de trabalho paulista. São Paulo: Editora Autores Associados, 2000.

23. JANNUZZI, Paulo M.; MONTAGNER, Paula; TAIRA, Edna. Migração e mobilidade social: revisitando a discussão a partir da PNAD 2014. Revista Ciências do Trabalho, n. 13, p. 1-15, 2019.

24. JESUS, Josimar G. Negros em movimento: migração e desigualdade racial no Brasil. 2020. Tese (Doutorado em Ciências - Economia Aplicada) - Escola Superior de Agricultura Luiz de Queiroz, Universidade de São Paulo, Piracicaba, 2020.

25. MAJOR, Lee E.; MACHIN, Stephen. Social mobility and its enemies. Londres: Penguin Books Limited, 2018.

26. OLIVEIRA, Camilla de. Mobilidade sócio-ocupacional no Brasil no novo milênio. Dissertação (Mestrado em Ciências - Economia Aplicada) - Escola Superior de Agricultura Luiz de Queiroz, Universidade de São Paulo, Piracicaba, 2018.

27. PARSONS, Talcott. An analytical approach to the theory of social stratification. In: PARSONS, Talcott. Essays in Sociological Theory. Ed. rev. Glencoe: Free Press, 1954. p. 69-88.

28. PASTORE, José. Desigualdade e mobilidade social no Brasil. São Paulo: TA Queiroz, 1979.

29. PASTORE, José; SILVA, Nelson V. Mobilidade social no Brasil. Makron books, 2000.

30. PASTORE, José; HALLER, Archibald O. O que está acontecendo com a mobilidade social no Brasil? In: ALBUQUERQUE, Roberto C. D.; VELLOSO, João Paulo D. R. Pobreza e mobilidade social: as bases do desenvolvimento moderno. São Paulo: Nobel, 1993. Cap. 1.

31. PAYNE, Geoff; ABBOTT, Pamela. The social mobility of women: Beyond male mobility models. Londres: Falmer, 1990.

32. RIBEIRO, Carlos A. C. Estrutura de classe e mobilidade social no Brasil. São Paulo: EDUSC, 2007.

33. RIBEIRO, Carlos A. C. Mobilidade e Estrutura de Classes no Brasil Contemporâneo. Sociologias, v. 16, n. 37, p. 178-217, 2014. https://doi. org/10.1590/15174522-016003711 
34. RIBEIRO, Carlos A. C. Tendências da desigualdade de oportunidades no Brasil: mobilidade social e estratificação educacional. Mercado de Trabalho: conjuntura e análise (IPEA), n. 62, p. 49-65, 2017.

35. ROGOFF, Natalie. Recent trends in occupational mobility. Nova York: The Free Press, 1953.

36. ROSE, David; MARSHALL, Gordon. Social Stratification. Madison: University of Wisconsin, Department of Sociology, 1988.

37. SALVATO, Márcio A.; SILVA, Denis G. O impacto da educação nos rendimentos do trabalhador: uma análise para região metropolitana de belo horizonte. In: Seminário sobre a Economia Mineira, XIII, 2008, Diamantina. Anais. Diamantina: Cedeplar-UFMG, 2008. p. 1-23.

38. SCALON, Maria C. Mobilidade social no Brasil: padrões e tendências. Editora Revan, 1999.

39. SILVA, Nelson V. Posição social das ocupações. Rio de Janeiro: IBGE, Centro de Informática, 1973.

40. SILVA, Nelson V. Mobilidade Social. In: MICELI, Sergio O. (Ed.). O que ler na ciência social brasileira (1970 - 1995). São Paulo: Editora Sumaré, 1999.

41. UEDA, Edric M. Educação e rendimentos: uma abordagem econométrica. 2001. Dissertação (Mestrado em Ciências Econômicas) - Universidade Estadual de Campinas, Campinas. 2001.

42. WRIGHT, Erik O. Class structure and income determination. Nova York: Academic Press, 1979.

43. YASUDA, Saburo. A methodological inquiry into social mobility. American Sociological Review, v. 29, n. 1, p. 16-23, 1964. https://doi.org/10.2307/2094637

Recebido em: 22 maio 2020.

Aceito em: 3 dez. 2020. 
